\title{
An (almost) unbiased estimator for the S-Gini index*
}

\author{
Thomas Demuynck ${ }^{\dagger}$
}

February 25, 2009

\begin{abstract}
This note provides an unbiased estimator for the absolute S-Gini and an almost unbiased estimator for the relative S-Gini for integer parameter values. Simulations indicate that these estimators perform considerably better then the usual estimators, especially for small sample sizes.
\end{abstract}

\section{The absolute and relative S-gini indices}

Assume that income is distributed according to a continuous and differentiable cumulative distribution function (cdf) $F:[0, \infty] \rightarrow[0,1]$ with finite mean, $\mu$, and continuous population density function (pdf) $f$. The absolute single-series Gini (absolute S-Gini), $A_{\infty}^{\delta}$, and the Relative single series Gini (relative S-Gini), $R_{\infty}^{\delta}$, with parameter $\delta \in \mathbb{R}_{++}$are given by:

$$
\begin{array}{r}
A_{\infty}^{\delta}=\mu-H_{\infty}^{\delta} \quad \text { and } \quad R_{\infty}^{\delta}=1-\frac{H_{\infty}^{\delta}}{\mu}, \\
\text { with } H_{\infty}^{\delta}=\delta \int_{0}^{\infty} x(1-F(x))^{\delta-1} d F(x) .
\end{array}
$$

These indices exist for all values of $\delta \geq 1$, but for values of $\delta<1$ it is possible that $H_{\infty}^{\delta}$ reaches infinity. From now on, we assume that $H_{\infty}^{\delta}$ is well defined for all values of $\delta$ under consideration.

The parameter $\delta$ determines the weight attached to the income of individuals at different points in the income distribution. As $\delta$ increases, more weight is given to the bottom of the income distribution. For $\delta$ equal to one, $H_{\infty}^{1}$ is equal to the mean $\mu$ and $R_{\infty}^{1}$ and $A_{\infty}^{1}$ are both equal to zero. For $\delta$ equal to 2, the indices $A_{\infty}^{2}$ and $R_{\infty}^{2}$ reduce to the well-known absolute and relative Ginis. We refer to Donaldson and Weymark (1980), Yitzhaki (1983) and Bossert (1990) for an in depth discussion of the properties related to the S-Gini index.

\footnotetext{
${ }^{*}$ I am pleased to acknowledge the insightful comments of Dirk Van de gaer.

†University of Ghent, Sherppa, Tweekerkenstraat 2, B-9000 Gent, Belgium. E-mail: thomas.demuynck@ugent.be
} 
The most common finite sample estimators for the S-Ginis are given by:

$$
\begin{array}{r}
A_{n}^{\delta}=\mu_{n}-H_{n}^{\delta} \\
\text { with } H_{n}^{\delta}=\frac{\sum_{i=1}^{n}\left((n-i+1)^{\delta}-(n-i)^{\delta}\right) \tilde{x}_{i}}{n^{\delta}}
\end{array}
$$

Here $\tilde{x}_{i}$ represents the $i$ th smallest value in the sample (the $i$ th order statistic) and $\mu_{n}$ is the sample mean, $\sum_{i=1}^{n} \tilde{x}_{i} / n$.

The estimators $A_{n}^{\delta}$ and $R_{n}^{\delta}$ are strongly consistent estimators for $A_{\infty}^{\delta}$ and $R_{\infty}^{\delta}$ and they are asymptotically normally distributed (Barrett and Pendakur, 1995; Zitikis and Gastwirth, 2002). Unfortunately, they are not unbiased and their bias depends on the sample size, $n$, the value of the parameter, $\delta$, and the distribution, $F$.

The sample mean $\mu_{n}$ is an unbiased estimator for the population mean $\mu$, hence, for the absolute S-Gini, $A_{\infty}^{\delta}$, we only need to construct an unbiased estimator for the term $H_{\infty}^{\delta}$. Such estimator would also provide us with an almost unbiased estimator for $R_{\infty}^{\delta}$. This last estimator is not unbiased because it is divided by the sample mean which is itself an estimator of the population mean. The next section provides an unbiased estimator of $H_{\infty}^{\delta}$ and the last section provides simulation results to compare these estimators with the estimators $A_{n}^{\delta}$ and $R_{n}^{\delta}$.

\section{A unbiased estimator for $H_{\infty}^{\delta}$}

We denote by $\left\{\begin{array}{l}n \\ k\end{array}\right\}$ the stirling number of the second kind with upper index $n$ and lower index $k$. The number $\left\{\begin{array}{l}n \\ k\end{array}\right\}$ represents the number of ways that a set of size $n$ can be partitioned into $k$ subsets. We denote by $\left(\begin{array}{l}n \\ k\end{array}\right)$ the binomial coefficient with upper index $n$ and lower index $k$, i.e. the number of $k$ element subsets of an $n$ element set. Finally, we denote by $\langle n\rangle_{k}$ the falling factorial $n(n-1) \ldots(n-k+1)$. The following identities ${ }^{1}$ will be used in this section:

$$
\begin{array}{rlr}
\left(\begin{array}{l}
n \\
k
\end{array}\right) & =\left(\begin{array}{c}
n \\
n-k
\end{array}\right), & \mathrm{R}-1 \\
\left\{\begin{array}{l}
n \\
k
\end{array}\right\} & =\left\{\begin{array}{l}
n-1 \\
k-1
\end{array}\right\}+k\left\{\begin{array}{c}
n-1 \\
k
\end{array}\right\}, & \mathrm{R}-2 \\
x^{r} & =\sum_{j=0}^{r}\left\{\begin{array}{l}
r \\
j
\end{array}\right\}\langle x\rangle_{j}, & \mathrm{R}-3
\end{array}
$$

\footnotetext{
${ }^{1}$ See Graham et al. (1989) for a proof of these identities.
} 


$$
\begin{aligned}
\langle k\rangle_{j}\left(\begin{array}{l}
n \\
k
\end{array}\right) & =\langle n\rangle_{j}\left(\begin{array}{l}
n-j \\
k-j
\end{array}\right), & \text { R-4 } \\
(x+y)^{n} & =\sum_{j=0}^{n}\left(\begin{array}{l}
n \\
j
\end{array}\right) x^{j} y^{n-j} . & \text { R-5 }
\end{aligned}
$$

We focus on the case where the parameter $\delta$ takes only integer values. Assume that we have a set of observations $\left\{x_{1}, \ldots, x_{n}\right\}$ that is drawn i.i.d. from the cdf $F$. The $i$ th order statistic $\tilde{x}_{i}$ will have pdf $f_{(i)}$ equal to:

$$
f_{(i)}(x)=i\left(\begin{array}{c}
n \\
i
\end{array}\right) F(x)^{i-1}(1-F(x))^{n-i} f(x)
$$

The expected value of $H_{n}^{\delta}$ equals:

$$
E\left(H_{n}^{\delta}\right)=\frac{1}{n^{\delta}} \sum_{i=1}^{n} i\left((n-i+1)^{\delta}-(n-i)^{\delta}\right)\left(\begin{array}{c}
n \\
i
\end{array}\right) \int_{0}^{\infty} x F(x)^{i-1}(1-F(x))^{n-i} d F(x)
$$

In order to simplify this expression we split it up into several parts:

$$
\begin{aligned}
E\left(H_{n}^{\delta}\right)= & \frac{1}{n^{\delta}} \int_{0}^{\infty} x \underbrace{\sum_{i=1}^{n} \underbrace{i(n-i+1)^{\delta}\left(\begin{array}{c}
n \\
i
\end{array}\right)}_{A_{1}} F(x)^{i-1}(1-F(x))^{n-i}-}_{A} \\
& \underbrace{\sum_{i=1}^{n} i(n-i)^{\delta}\left(\begin{array}{c}
n \\
i
\end{array}\right)}_{B=1} F(x)^{i-1}(1-F(x))^{n-i} d F(x) .
\end{aligned}
$$

We have that:

$$
\begin{array}{rlrl}
A_{1} & =i(n-i+1)^{\delta}\left(\begin{array}{c}
n \\
i
\end{array}\right) & (\mathrm{R}-4) & B_{1}=i(n-i)^{\delta}\left(\begin{array}{c}
n \\
i
\end{array}\right) \\
& =n(n-i+1)^{\delta}\left(\begin{array}{c}
n-1 \\
i-1
\end{array}\right) & =n(n-i)^{\delta}\left(\begin{array}{c}
n-1 \\
i-1
\end{array}\right) \\
& =(n-i+1)^{\delta+1}\left(\begin{array}{c}
n \\
n-i+1
\end{array}\right) & & =\sum_{j=0}^{\delta} n\left\{\begin{array}{l}
\delta \\
j
\end{array}\right\}\langle n-i\rangle_{j}\left(\begin{array}{c}
n-1 \\
n-i
\end{array}\right) \\
& =\sum_{j=0}^{\delta+1}\left\{\begin{array}{c}
\delta+1 \\
j
\end{array}\right\}\langle n\rangle_{j}\left(\begin{array}{c}
n-j \\
n-i-j+1
\end{array}\right)(\mathrm{R}-3, \mathrm{R}-4) & & =\sum_{j=0}^{\delta}\left\{\begin{array}{l}
\delta \\
j
\end{array}\right\}\langle n\rangle_{j+1}\left(\begin{array}{c}
n-1-j \\
i-1
\end{array}\right) .
\end{array}
$$




$$
=\sum_{j=1}^{\delta+1}\left\{\begin{array}{c}
\delta+1 \\
j
\end{array}\right\}\langle n\rangle_{j}\left(\begin{array}{c}
n-j \\
i-1
\end{array}\right) .
$$

These results enable us to simplify $A$ and $B$ :

$$
\begin{aligned}
A & =\sum_{i=1}^{n} \sum_{j=1}^{\delta+1}\left\{\begin{array}{c}
\delta+1 \\
j
\end{array}\right\}\langle n\rangle_{j}\left(\begin{array}{c}
n-j \\
i-1
\end{array}\right) F(x)^{i-1}(1-F(x))^{n-i} \\
& =\sum_{j=1}^{\delta+1}\left\{\begin{array}{c}
\delta+1 \\
j
\end{array}\right\}\langle n\rangle_{j} \sum_{i=1}^{n}\left(\begin{array}{c}
n-j \\
i-1
\end{array}\right) F(x)^{i-1}(1-F(x))^{n-i} \\
& =\sum_{j=1}^{\delta+1}\left\{\begin{array}{c}
\delta+1 \\
j
\end{array}\right\}\langle n\rangle_{j}(1-F(x))^{j-1} . \\
B & =\sum_{i=1}^{n} \sum_{j=0}^{\delta}\left\{\begin{array}{c}
\delta \\
j
\end{array}\right\}\langle n\rangle_{j+1}\left(\begin{array}{c}
n-1-j \\
i-1
\end{array}\right) F(x)^{i-1}(1-F(x))^{n-i} \\
& =\sum_{j=0}^{\delta}\left\{\begin{array}{c}
\delta \\
j
\end{array}\right\}\langle n\rangle_{j+1} \sum_{i=1}^{n}\left(\begin{array}{c}
n-1-j \\
i-1
\end{array}\right) F(x)^{i-1}(1-F(x))^{n-i} \\
& =\sum_{j=0}^{\delta}\left\{\begin{array}{l}
\delta \\
j
\end{array}\right\}\langle n\rangle_{j+1}(1-F(x))^{j} \\
& =\sum_{j=1}^{\delta+1}\left\{\begin{array}{c}
\delta \\
j-1
\end{array}\right\}\langle n\rangle_{j}(1-F(x))^{j-1} .
\end{aligned}
$$

Substituting $A$ and $B$ into equation (1) gives:

$$
\begin{aligned}
E\left(H_{n}^{\delta}\right) & =\frac{1}{n^{\delta}} \int_{-\infty}^{\infty} x \sum_{j=1}^{\delta+1}\left(\left\{\begin{array}{c}
\delta+1 \\
j
\end{array}\right\}-\left\{\begin{array}{c}
\delta \\
j-1
\end{array}\right\}\right)\langle n\rangle_{j}(1-F)^{j-1} d F(x) \\
& =\frac{1}{n^{\delta}} \int_{-\infty}^{\infty} x \sum_{j=1}^{\delta+1} j\left\{\begin{array}{l}
\delta \\
j
\end{array}\right\}\langle n\rangle_{j}(1-F)^{j-1} d F(x) \\
& =\frac{1}{n^{\delta}} \sum_{j=1}^{\delta}\left\{\begin{array}{l}
\delta \\
j
\end{array}\right\}\langle n\rangle_{j} H_{\infty}^{j} .
\end{aligned}
$$

Equation (2) shows that the expected value of $H_{n}^{\delta}$ can be expressed as a weighted average of all indices $H_{\infty}^{m}$ with $m \leq \delta$. As such, the estimator $H_{n}^{\delta}$ will not be unbiased unless $H_{\infty}^{m}$ 
is zero for all $m \leq \delta$. Equation 2 allows us to construct an unbiased estimator of $H_{\infty}^{\delta}$ in a recursive way.

For $\delta=1$, we have that $E\left(H_{n}^{1}\right)=H_{\infty}^{1}=\mu$. Hence, $H_{n}^{1}$ is an unbiased estimator of $H_{\infty}^{1}$. Now, assume that we have an unbiased estimator $h_{n}^{m}$ of $H_{\infty}^{m}$ for all $m$ in $\{1,2, \ldots, \delta-1\}$. Then we can construct following estimator $h_{n}^{\delta}$ of $H_{\infty}^{\delta}$ :

$$
h_{n}^{\delta}=\frac{1}{\langle n\rangle_{\delta}}\left(n^{\delta} H_{n}^{\delta}-\sum_{j=0}^{\delta-1}\left\{\begin{array}{l}
\delta \\
j
\end{array}\right\}\langle n\rangle_{j} h_{n}^{j}\right)
$$

This estimator is unbiased:

$$
\begin{aligned}
E\left(h_{n}^{\delta}\right) & =E\left(\frac{1}{\langle n\rangle_{\delta}}\left(n^{\delta} H_{n}^{\delta}-\sum_{j=0}^{\delta-1}\left\{\begin{array}{l}
\delta \\
j
\end{array}\right\}\langle n\rangle_{j} h_{n}^{j}\right)\right) \\
& =\frac{1}{\langle n\rangle_{\delta}}\left(n^{\delta} E\left(H_{n}^{\delta}\right)-\sum_{j=0}^{\delta-1}\left\{\begin{array}{l}
\delta \\
j
\end{array}\right\}\langle n\rangle_{j} E\left(h_{n}^{j}\right)\right) \\
& =H_{\infty}^{\delta} .
\end{aligned}
$$

The unbiased estimator for $A_{\infty}^{\delta}$ is then given by $a_{n}^{\delta}=\mu_{n}-h_{n}^{\delta}$ and the almost unbiased estimator for $R_{\infty}^{\delta}$ is given by $r_{n}^{\delta}=1-h_{n}^{\delta} / \mu_{n}$. For the Gini index, i.e. $\delta=2$, it can be shown that $r_{n}^{2}=n R_{n}^{\delta} /(n-1)$. This is in agreement to the first order correction for the Gini index found in the literature (see Deaton, 1997; Deltas, 2003; Davidson, 2009). It can be shown that $h_{n}^{\delta}$ is equal to the following expression ${ }^{2}$ :

$$
h_{n}^{\delta}=\sum_{i=1}^{n} \frac{\delta\langle n-i\rangle_{\delta-1}}{\langle n\rangle_{\delta}} \tilde{x}_{i}
$$

The multiplicators $\delta\langle n-i\rangle_{\delta-1} /\langle n\rangle_{\delta}$ sum to one ${ }^{3}$ which implies that, analogue to the estimators $H_{n}^{\delta}$, the estimators $h_{n}^{\delta}$ are a weighted average of the order statistics $\tilde{x}_{i}$. Also, note that the weights attached to the $\delta-1$ highest incomes are equal to zero. This implies that the estimator $h_{n}^{\delta}$ does not use all available information. For example, the value of $h_{n}^{10}$ on a sample of size 10 coincides with the smallest value in the sample.

Simple manipulation of equation (4) shows that we can write $h_{n}^{\delta}$ as $\sum_{i=1}^{n} a_{i} \tilde{x}_{i}$, with

$$
a_{i}=\left\{\begin{array}{l}
\delta / n \quad \text { if } i=1 \\
a_{i-1}\left(1-\frac{\delta-1}{n-(i-1)}\right) \text { for } i>1
\end{array}\right.
$$

\footnotetext{
${ }^{2}$ See appendix A.

${ }^{3}$ See appendix B
} 
For $\delta \geq 1$, as $i$ increases, the weights attached to $\tilde{x}_{i}$ decrease in an increasing rate until they reach zero for $\tilde{x}_{n-\delta+2}$. The recursion (5) shows that the estimator $h_{n}^{\delta}$ is very easy to calculate. It also makes it possible to define $h_{n}^{\delta}$ for non-integer values of $\delta$. Unfortunately, this extension has the unwanted side-effects that the weights $a_{i}$ do no longer sum to unity, although is will approximate unity if $n$ is not to small, and that the estimator is no longer unbiased.

\section{Simulation}

For our empirical illustration we used a lognormal distribution with parameters 9.85 and 0.6. Our population statistics $A_{\infty}^{\delta}$ and $R_{\infty}^{\delta}$ were calculated on the basis of a random sample of 50 million observations. We drew 200.000 independent samples of size $\mathrm{m}(m=10,30,50)$. For each of these samples, we calculated the estimators $A_{m}^{\delta}, a_{n}^{\delta}, R_{m}^{\delta}$ and $r_{m}^{\delta}$. Table 1 presents the averages over these 200.000 samples (standard errors are between brackets) for the values $\delta=1.5 ; 2 ; 5 ; 7.5$ and 10 . Simulation results for other parameter values and other distributions give similar results.

Table 1: simulation results

\begin{tabular}{|c|c|c|c|c|c|c|c|}
\hline$\overline{\bar{\delta}}$ & $\begin{array}{c}\text { sample } \\
\text { size }\end{array}$ & $\overline{A_{n}^{\delta}}$ & $a_{n}^{\delta}$ & $\overline{A_{\infty}^{\delta}}$ & $\overline{R_{n}^{\delta}}$ & $r_{n}^{\delta}$ & $R_{\infty}^{\delta}$ \\
\hline \multirow[t]{3}{*}{1.5} & 10 & $\begin{array}{l}4296 \\
(1764)\end{array}$ & $\begin{array}{l}4378 \\
(1852)\end{array}$ & \multirow{3}{*}{4941} & $\begin{array}{c}0.1853 \\
(0.0495)\end{array}$ & $\begin{array}{l}0.1886 \\
(0.0530)\end{array}$ & \multirow{3}{*}{0.2177} \\
\hline & 30 & $\begin{array}{l}4708 \\
(1115)\end{array}$ & $\begin{array}{l}4802 \\
(1146)\end{array}$ & & $\begin{array}{c}0.2059 \\
(0.033)\end{array}$ & $\begin{array}{c}0.2100 \\
(0.034)\end{array}$ & \\
\hline & 50 & $\begin{array}{l}4799 \\
(882)\end{array}$ & $\begin{array}{c}4870 \\
(899) \\
\end{array}$ & & $\begin{array}{c}0.2105 \\
(0.0262)\end{array}$ & $\begin{array}{l}0.2136 \\
(0.0268)\end{array}$ & \\
\hline \multirow[t]{3}{*}{2} & 10 & $\begin{array}{l}6733 \\
(2600)\end{array}$ & $\begin{array}{l}7481 \\
(2890)\end{array}$ & \multirow{3}{*}{7458} & $\begin{array}{l}0.2908 \\
(0.0696)\end{array}$ & $\begin{array}{l}0.3231 \\
(0.0774)\end{array}$ & \multirow{3}{*}{0.3286} \\
\hline & 30 & $\begin{array}{l}7217 \\
(1574)\end{array}$ & $\begin{array}{l}7466 \\
(1628)\end{array}$ & & $\begin{array}{l}0.3158 \\
(0.0431)\end{array}$ & $\begin{array}{l}0.3267 \\
(0.0446)\end{array}$ & \\
\hline & 50 & $\begin{array}{l}7307 \\
(1227)\end{array}$ & $\begin{array}{l}7455 \\
(1252)\end{array}$ & & $\begin{array}{l}0.3207 \\
(0.0344)\end{array}$ & $\begin{array}{c}0.3273 \\
(0.0347)\end{array}$ & \\
\hline \multirow[t]{3}{*}{5} & 10 & $\begin{array}{c}11545 \\
(3837)\end{array}$ & $\begin{array}{c}12515 \\
(4055)\end{array}$ & \multirow{3}{*}{12505} & $\begin{array}{c}0.5011 \\
(0.0938)\end{array}$ & $\begin{array}{l}0.5438 \\
(0.0982)\end{array}$ & \multirow{3}{*}{0.5508} \\
\hline & 30 & $\begin{array}{c}12193 \\
(2250)\end{array}$ & $\begin{array}{c}12509 \\
(2289)\end{array}$ & & $\begin{array}{l}0.5345 \\
(0.0535)\end{array}$ & $\begin{array}{c}0.5484 \\
(0.0541)\end{array}$ & \\
\hline & 50 & $\begin{array}{c}12319 \\
(1751)\end{array}$ & $\begin{array}{c}12508 \\
(1768)\end{array}$ & & $\begin{array}{l}0.5411 \\
(0.0415)\end{array}$ & $\begin{array}{c}0.5494 \\
(0.0417)\end{array}$ & \\
\hline \multirow[t]{3}{*}{7.5} & 10 & $\begin{array}{c}12729 \\
(4082)\end{array}$ & $\begin{array}{c}13853 \\
(4346)\end{array}$ & \multirow{3}{*}{13894} & $\begin{array}{c}0.5545 \\
(0.0993)\end{array}$ & $\begin{array}{l}0.6043 \\
(0.1065)\end{array}$ & \multirow{3}{*}{0.6123} \\
\hline & 30 & $\begin{array}{c}13526 \\
(2395)\end{array}$ & $\begin{array}{c}13900 \\
(2438)\end{array}$ & & $\begin{array}{l}0.5936 \\
(0.0556)\end{array}$ & $\begin{array}{l}0.6101 \\
(0.0563)\end{array}$ & \\
\hline & 50 & $\begin{array}{c}13671 \\
(1863)\end{array}$ & $\begin{array}{c}13895 \\
(1882)\end{array}$ & & $\begin{array}{c}0.60113 \\
(0.0428)\end{array}$ & $\begin{array}{c}0.61098 \\
(0.0430)\end{array}$ & \\
\hline \multirow[t]{2}{*}{10} & 10 & $\begin{array}{c}13398 \\
(4246)\end{array}$ & $\begin{array}{c}14722 \\
(4598)\end{array}$ & & $\begin{array}{l}0.5828 \\
(0.1026)\end{array}$ & $\begin{array}{l}0.6414 \\
(0.1151)\end{array}$ & \multirow[b]{2}{*}{0.6480} \\
\hline & 30 & $\begin{array}{c}14287 \\
(2488)\end{array}$ & $\begin{array}{c}14715 \\
(2539)\end{array}$ & 14706 & $\begin{array}{l}0.6268 \\
(0.0569)\end{array}$ & $\begin{array}{l}0.6457 \\
(0.0580)\end{array}$ & \\
\hline
\end{tabular}


Table 1: simulation results

\begin{tabular}{cc|ccc||ccc}
\hline \hline$\delta$ & $\begin{array}{c}\text { sample } \\
\text { size }\end{array}$ & $A_{n}^{\delta}$ & $a_{n}^{\delta}$ & $A_{\infty}^{\delta}$ & $R_{n}^{\delta}$ & $r_{n}^{\delta}$ & $R_{\infty}^{\delta}$ \\
\hline 50 & $\begin{array}{c}14443 \\
(1942)\end{array}$ & $\begin{array}{c}14698 \\
(1947)\end{array}$ & & $\begin{array}{c}0.6353 \\
(0.0437)\end{array}$ & $\begin{array}{c}0.6466 \\
(0.0441)\end{array}$ &
\end{tabular}

NOTE: These simulations were based on the lognormal distribution: $\ln X \sim N(9.85,0.6)$. The statistics $R_{\infty}^{\delta}$ and $A_{\infty}^{\delta}$ were based on a random sample of 10 million observations. Each average was computed over a set of 200.000 samples. Standard errors are between brackets.

We observe following regularities:

- For integer parameter values, the estimators $r_{n}^{\delta}$ and $a_{n}^{\delta}$ performs considerably better then the estimators $R_{n}^{\delta}$ and $A_{n}^{\delta}$.

- For noninteger parameter values one can clearly see that the estimator $a_{n}^{\delta}$ is no longer unbiased although the bias decreases for larger sample sizes and larger parameter values. Furthermore, the estimators $r_{n}^{\delta}$ and $a_{n}^{\delta}$ seem to perform considerably better in comparison to the estimators $A_{n}^{\delta}$ and $R_{n}^{\delta}$.

- The standard errors for the estimators $r_{n}^{\delta}$ and $a_{n}^{\delta}$ are slightly larger compared to the standard errors for the estimators $R_{n}^{\delta}$ and $A_{n}^{\delta}$.

\section{References}

Barrett, G. F., Pendakur, K., 1995. The asymptotic distribution of the generalized gini indices of inequality. Canadian Journal of Economics 28, 1042-1055.

Bossert, W., 1990. An axiomatization of the single-series ginis. Journal of Economic Theory $50,82-92$.

Davidson, R., 2009. Reliable inference for the gini index. GREQAM Document de Travail nr 2007-23.

Deaton, A. S., 1997. The analysis of household surveys: a microeconometric approach to development policy. John Hopkins University Press for the World Bank, Baltimore.

Deltas, G., 2003. The small-sample bias of the gini coefficient: results and implications for empirical research. The Review of Economics and Statistics 85, 226-234.

Donaldson, D., Weymark, J. A., 1980. A single-parameter generalization of the gini indices of inequality. Journal of Economic Theory 22, 67-86.

Graham, R. L., Knuth, D. E., Patashnik, O., 1989. Concrete Mathematics. Addison-Wesley.

Yitzhaki, S., 1983. Relative deprivation and the gini coefficient. International Economic Review 93, 617-628. 
Zitikis, R., Gastwirth, J., 2002. The asymptotic distribution of the s-gini index. Australian and New Zealand Journal of Statistics 44, 439-446.

\section{A Equivalence of equation 3 and 4}

The proof is by induction on $\delta$. For $\delta=1$ we easily establish that both equations 3 and 4 reduce to $\mu_{n}$. Assume that the assertion holds for all $m<\delta$. The proof follows if we can show that:

$$
n^{\delta} H_{n}^{\delta}=\sum_{j=0}^{\delta}\left\{\begin{array}{l}
\delta \\
j
\end{array}\right\}\langle n\rangle_{j} h_{n}^{j} .
$$

where $h_{n}^{j}$ is given by equation 4 .

$$
\begin{aligned}
n^{\delta} H_{n}^{\delta} & =\sum_{i=1}^{n}(n-i+1)^{\delta}-(n-i)^{\delta} \tilde{x}_{i} \\
& =\sum_{i=1}^{\delta} \tilde{x}_{i} \sum_{j=0}^{\delta}\left\{\begin{array}{l}
\delta \\
j
\end{array}\right\}\langle n-i+1\rangle_{j}-\sum_{i=1}^{n} \tilde{x}_{i} \sum_{j=0}^{\delta}\left\{\begin{array}{l}
\delta \\
j
\end{array}\right\}\langle n-i\rangle_{j} \\
& =\sum_{i=1}^{n} \tilde{x}_{i} \sum_{j=0}^{\delta}\left\{\begin{array}{l}
\delta \\
j
\end{array}\right\}\langle n-i\rangle_{j-1}((n-i+1)-(n-i-j+1)) \\
& =\sum_{i=1}^{n} \tilde{x}_{i} \sum_{j=0}^{\delta}\left\{\begin{array}{l}
\delta \\
j
\end{array}\right\} j\langle n-i\rangle_{j-1} \\
& =\sum_{i=1}^{n} \sum_{j=0}^{\delta}\left\{\begin{array}{l}
\delta \\
j
\end{array}\right\}\langle n\rangle_{j} \frac{j\langle n-i\rangle_{j-1}}{\langle n\rangle_{j}} \tilde{x}_{i} \\
& =\sum_{j=0}^{\delta}\left\{\begin{array}{l}
\delta \\
j
\end{array}\right\}\langle n\rangle_{j} h_{n}^{j} .
\end{aligned}
$$




\section{B $\quad h_{n}^{\delta}$ is a weighted sum}

We show that the weights $\frac{j\langle n-i\rangle_{j-1}}{\langle n\rangle_{j}}$ sum to one.

$$
\begin{aligned}
\sum_{i=1}^{n} \frac{j\langle n-i\rangle_{j-1}}{\langle n\rangle_{j}} & =\sum_{i=1}^{n} \frac{j}{n} \frac{(n-j) !}{(i-1) !(n-i-j+1) !} \frac{(i-1) !(n-i) !}{(n-1) !} \\
& =\frac{j}{n} \sum_{i=1}^{n}\left(\begin{array}{c}
n-j \\
i-1
\end{array}\right) /\left(\begin{array}{c}
n-1 \\
i-1
\end{array}\right) \\
& =\frac{j}{n} \sum_{k=0}^{n-1}\left(\begin{array}{c}
n-j \\
k
\end{array}\right) /\left(\begin{array}{c}
n-1 \\
k
\end{array}\right) \\
& =1 .
\end{aligned}
$$

The last step uses the identity: $\sum_{k=0}^{m}\left(\begin{array}{l}n \\ k\end{array}\right) /\left(\begin{array}{c}m \\ k\end{array}\right)=\frac{m+1}{m+1-n}$ (see Graham et al., 1989, problem 1, p173). 